Article

\title{
In-plane Switching Deformed Helix Ferroelectric Liquid Crystal Display Cells
}

\section{Evgeny P. Pozhidaev *, Timofey P. Tkachenko, Artemy V. Kuznetsov and Igor N. Kompanets}

P. N. Lebedev Physical Institute of RAS, 53 Leninskiy Prospekt, Moscow 119991, Russia; tptkachenko@lebedev.ru (T.P.T.); tem4rus@gmail.com (A.V.K.); kompanecin@lebedev.ru (I.N.K.)

* Correspondence: epozhidaev@mail.ru; Tel.: +7-903-5260935

Received: 9 October 2019; Accepted: 19 October 2019; Published: 21 October 2019

\begin{abstract}
In-plane electro-optical switching (IPS) is a natural feature of a conventional planar-aligned display cell based on the deformed helix ferroelectric liquid crystal effect (DHFLC-effect) with a sub-wavelength helix pitch, if the tilt angle is close to 40 degrees.
\end{abstract}

Keywords: deformed helix ferroelectric liquid crystal effect; in-plane switching

\section{Introduction}

It is well known that the principal advantage of in-plane switching (IPS) nematic liquid crystal (NLC) display cells [1-3] is their color accuracy due to small gamma and color shifts. And, since the principal axes of the NLC layer always remains in the substrates plane in the process of switching, the viewing angle is large and symmetric [4]. Together with this, the production of IPS displays based on nematic liquid crystals is associated with the solution of rather complex technological problems caused by the need to form a grid of interdigitated electrodes (Figure 1).
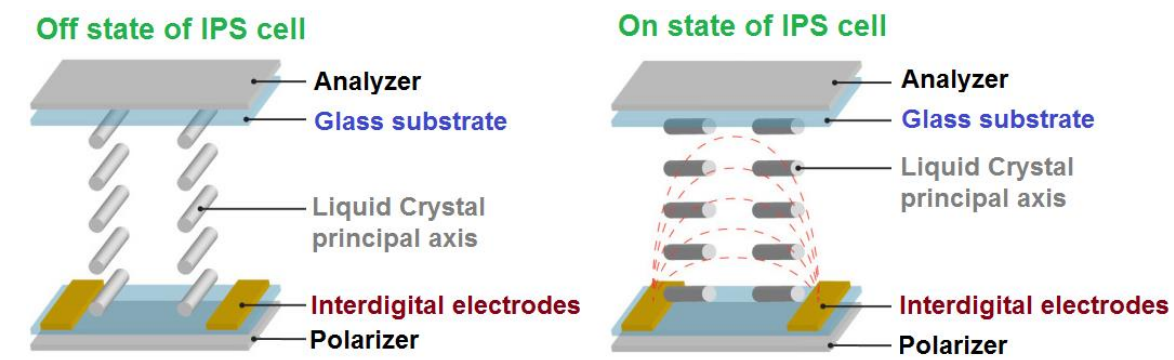

Figure 1. Off and on states of an in-plane switching (IPS) nematic liquid crystal (NLC) cell. Red dashes show the lines of force of the electric field $\boldsymbol{E}$.

We show further that the in-plane switching effect is observed not only with NLC cells, but also in planar-aligned deformed helix ferroelectric liquid crystal (DHFLC) cells [5]. The simple manufacturing techniques of planar-aligned DHFLCs, where continuous but not interdigitated electrodes are in use (Figure 2), are well mastered, and the electro-optical modulation in these structures has been studied in detail both experimentally and theoretically [6-9]. 


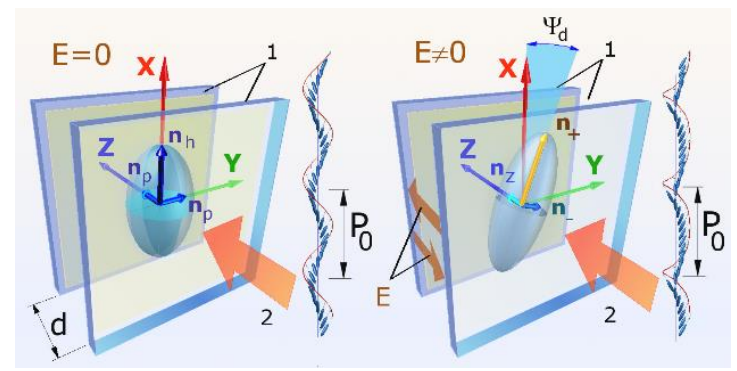

Figure 2. Geometry of a planar oriented deformed helix ferroelectric liquid crystal (DHFLC) cell with a uniform lying helix: Number 1 designates glass plates coated with continuous indium tin oxide (ITO) layers, over which the aligning layers are deposited; Arrow 2 shows the direction of propagation of linearly polarized incident light; $d$ is the DHFLC layer thickness; $p_{0}$ is the helix pitch, $n_{p}$ and $n_{h}$ are refractive indices of the DHFLC helical structure at $E=0$ [10]; and $\Psi_{d}(E) \sim E$ is the deflection of the main optical axis in the electric field.

Replacing the periodical helical nanostructure of DHFLC cells with macroscopic ellipsoids of effective refractive indices (Figure 2) when analyzing light propagation through the structure will be quite correct at a certain wavelength $\lambda$, despite some difficulties related to effects of optical rotation and Bragg reflection, if [11]

$$
p_{0} \leq \lambda / 5
$$

Figure 2 illustrates the specific biaxial transformation of the ellipsoid of effective refractive indices $n_{+} \sim E^{2}, n_{-} \sim E^{2}$ and $n_{z} \sim E^{2}$ in electric field $E$ in combination with the main optical axis deflection on the angle $\Psi_{d} \sim E$ [6-10], which occurs perpendicular to the electric field direction [10]; that is, in the plane of the substrates (indicated by the number 1 in Figure 2). Thus, there is some analogy between switching the main optical axis in IPS NLC (Figure 1) and planar-aligned DHFLC (Figure 2) cells. Consequently, the IPS effect in DHFLC cells is possible if the mentioned above biaxial transformation of effective refractive indices does not significantly affect the propagation of light in the DHFLC layer. This paper is devoted to experimental proof of the existence of such a possibility. Our approach is based on the fact that when condition (1) is fulfilled, the light transmission $T$ of a planar-aligned DHFLC layer located between crossed polarizers is described by the relation proved both theoretically and experimentally [7]:

$$
T(E)=\sin ^{2} 4 \Psi_{d}(E) \cdot \sin ^{2} \frac{\pi \Delta n_{e f f}(E) d}{\lambda},
$$

where $\Delta n_{e f f}(E)=n_{+}(E)-n_{-}(E)$ is the effective birefringence of the DHFLC layer in electric field.

\section{Experimental Results and Discussion}

Two multicomponent ferroelectric liquid crystal (FLC) mixtures with a sub-wavelength helix pitch $\left(p_{0}<100 \mathrm{~nm}\right)$ were elaborated by the authors for investigations of IPS switching in DHFLC electrooptical cells. These are FLC-587-F7 possessing the tilt angle $\theta=31.6^{\circ}$ at $22^{\circ} \mathrm{C}$ and FLC-650 $\left(\theta=38.4^{\circ}\right.$ at $22^{\circ} \mathrm{C}$, as shown in Figure 3). Both nano-scale helix pitch FLC mixtures are composed of biphenylpyrimidines as an achiral smectic $\mathrm{C}$ matrix and trifluoromethylalkyl diesters of p-terphenyldicarboxylic acid as chiral dopants possessing very high helical twisting power, as it has been described in detail [12]. These mixtures, which are special cases of the general material science approach described in [12], were taken into consideration, since they are characterized by rather different dependencies $\Delta n_{\text {eff }}(E)$ and $\Psi_{d}(E)$, see Figure 3. 


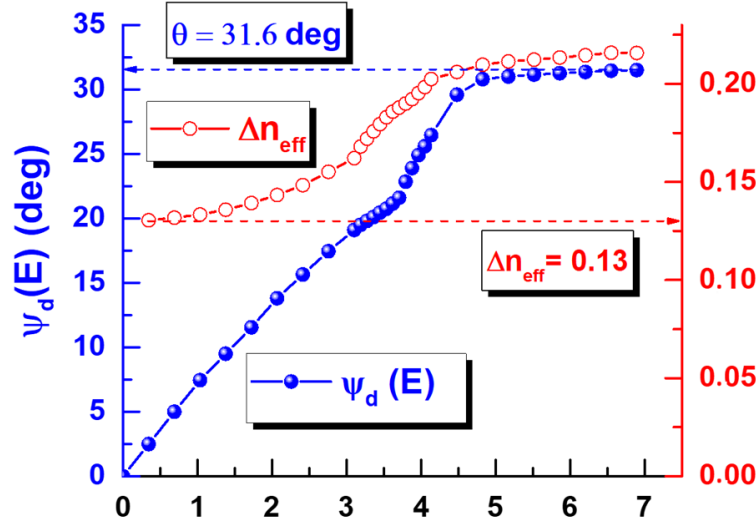

(a)

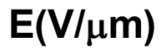

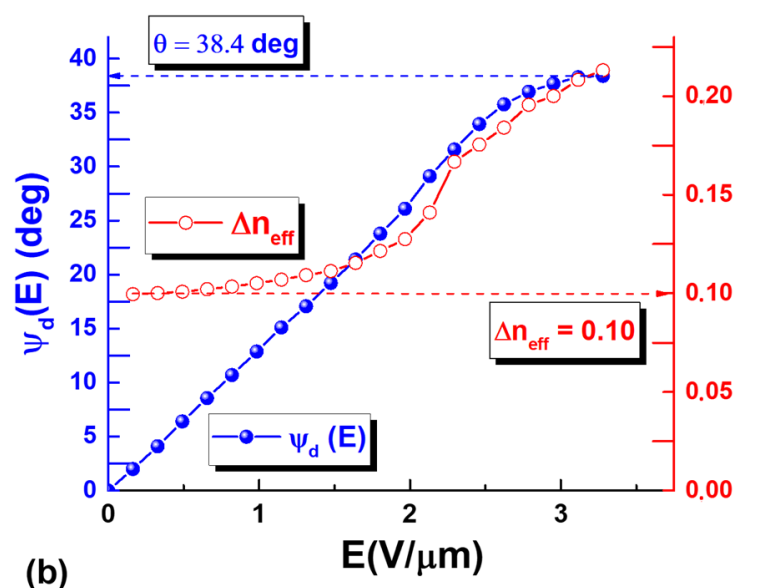

(b)

Figure 3. Dependencies of $\Delta n_{\text {eff }}(E)$ (red circles) and $\Psi_{d}(E)$ (blue balls) that on the left (a) relates to the FLC-587-F7, and on the right (b) to the FLC-650. Measurements were carried out at $22{ }^{\circ} \mathrm{C}$, at a wavelength of $\lambda=632.8 \mathrm{~nm}$.

The above dependencies were obtained in the experimental setup based on the scheme of the Mach-Zehnder interferometer, which is described in detail [8]. Note that the quantitative value of the tilt angle $\theta$ of molecules in smectic layers in helicoidal structures of FLCs is determined by the level of saturation of the $\Psi_{d}(E)$ experimental curve, whose behavior in the electric field (as well as $\Delta n_{\text {eff }}(E)$ ) depends on the tilt angle magnitude, as can be seen in Figure 3.

Figure 4 shows measured $T(E)$ dependencies of two planar aligned DHFLC cells (filled with the FLC-587-F7 on the left and with the FLC-650 on the right) and calculated values of the factors included in the relation (2). Measured dependencies of $\Psi_{d}(E)$ and $\Delta n_{e f f}(E)$, which are presented in Figure 3, were taken for the calculations.
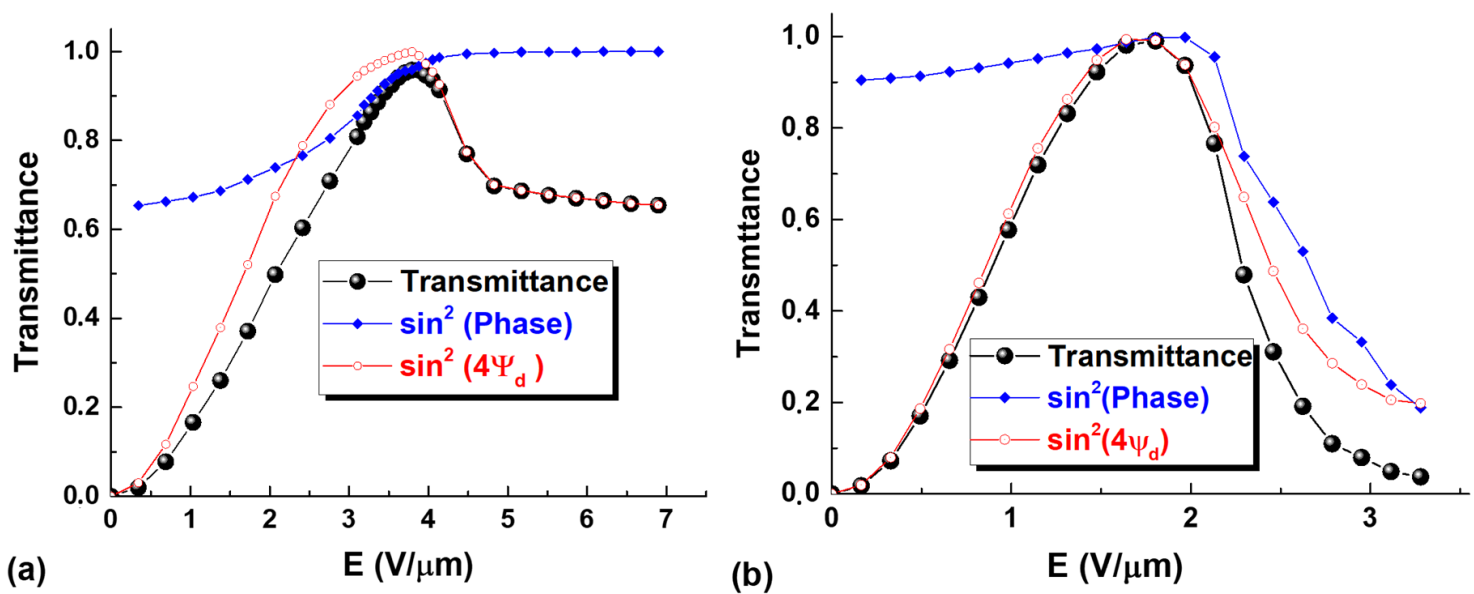

Figure 4. (a) Measured total light transmittance $T(E)$ (black balls) and calculated values of the factors included in the relation (2) for the DHFLC cell filled with the FLC-587-F7 at $\mathrm{d}=1.45 \mu \mathrm{m}$ and placed between crossed polarizers; (b) the same data but for the FLC-650 at $\mathrm{d}=3.05 \mu \mathrm{m}$. Calculated components of the transmittance are shown by red circles and blue diamonds. Measurements were carried out at $22{ }^{\circ} \mathrm{C}$, at a wavelength of $\lambda=632.8 \mathrm{~nm}$.

Since the measured $T(E)$ dependence in Figure $4 \mathrm{~b}$ (black balls) is practically coinciding with the calculated dependence $\sin ^{2}\left(4 \Psi_{d}\right)$, and the factor $\sin ^{2}\left(\pi \Delta \mathrm{n}_{\text {eff }} \mathrm{d} / \lambda\right)$, which is denoted as $\sin ^{2}$ (Phase) in the figure, practically does not change when $0 \leq T(E) \leq 1$; it can be argued that in this case the electrooptical modulation is very close to in-plane switching. However, the same factor in Figure 4a changes by $45 \%$ when $0 \leq T(E) \leq 1$, which is not very consistent with the definition of the IPS-mode. Thus, the 
electro-optical modulation in the planar-aligned DHFLC cells approaches the in-plane switching mode as the $\theta$ angle increases, which can be seen when comparing Figure 3; Figure 4.

\section{Conclusions}

It is experimentally shown that the in-plane switching electrooptical mode can be observed in DHFLC cells with the sub-wavelength helix pitch. Planar-aligned DHFLC IPS cells have a technological advantage compared with NLC IPS cells, since for manufacturing of DHFLC cells continuous and not interdigitated electrodes are in use. In addition, any DHFLC cells provide electro-optical modulation in the kilohertz range $[6,12]$, which is also their obvious advantage over IPS NLC.

Author Contributions: Methodology, material science and writing—original draft preparation, E.P.P.; experiment, T.P.T. and A.V.K.; supervision, I.N.K.

Funding: This research was funded by the Russian Ministry of Science \& Higher Education, the unique project identifier RFMEFI60417X0191.

Acknowledgments: The authors acknowledge V. V. Kesaev for technical help and fruitful discussions.

Conflicts of Interest: The authors declare no conflict of interest.

\section{References}

1. Oh-E, M.; Kondo, K. Electro-optical characteristics and switching behavior of the in-plane switching mode. Appl. Phys. Lett. 1995, 67, 3895. [CrossRef]

2. Oh-E, M.; Kondo, K. Response mechanism of nematic liquid crystals using the in-plane switching mode. Appl. Phys. Lett. 1996, 69, 623. [CrossRef]

3. Oh-E, M.; Kondo, K. The in-plane switching of homogeneously aligned nematic liquid crystals. Liq. Cryst. 1997, 22, 379. [CrossRef]

4. Oh-E, M.; Yoneya, M.; Ohta, M.; Kondo, K. Dependence of viewing angle characteristics on pretilt angle in the in-plane switching mode. Liq. Cryst. 1997, 22, 391. [CrossRef]

5. Beresnev, L.A.; Chigrinov, V.G.; Dergachev, D.I.; Pozhidaev, E.P.; Funfshilling, J.; Schadt, M. Deformed helix ferroelectric liquid crystal display-A new electrooptic mode in ferroelectric smectic $C^{*}$ liquid crystals. Liq. Cryst. 1989, 5, 1171. [CrossRef]

6. Pozhidaev, E.; Torgova, S.; Minchenko, M.; Yednak, C.A.R.; Strigazzi, A.; Miraldi, E. Phase modulation and ellipticity of the light transmitted through a smectic $C^{*}$ layer with short helix pitch. Liq. Cryst. 2010, 37, 1067. [CrossRef]

7. Kiselev, A.D.; Pozhidaev, E.P.; Chigrinov, V.G.; Kwok, H.S. Polarization-gratings approach to deformed-helix ferroelectric liquid crystals with subwavelength pitch. Phys. Rev. E 2011, 83, 031703. [CrossRef] [PubMed]

8. Kotova, S.P.; Samagin, S.A.; Pozhidaev, E.P.; Kiselev, A.D. Light modulation in planar aligned short-pitch deformed-helix ferroelectric liquid crystals. Phys. Rev. E 2015, 92, 062502. [CrossRef] [PubMed]

9. Kesaev, V.V.; Kiselev, A.D.; Pozhidaev, E.P. Modulation of unpolarized light in planar-aligned subwavelength-pitch deformed-helix ferroelectric liquid crystals. Phys. Rev. E 2017, 95, 032705.

10. Pozhidaev, E.P.; Schrivastava, A.K.; Kiselev, A.D.; Chigrinov, V.G.; Vashchenko, V.V.; Krivoshey, A.I.; Minchenko, M.V.; Kwok, H.S. Enhanced orientational Kerr effect in vertically aligned deformed helix ferroelectric liquid crystals. Opt. Lett. 2014, 39, 2900. [CrossRef] [PubMed]

11. Hubert, P.; Jägemalm, P.; Oldano, C.; Rajteri, M. Optic models for short-pitch cholesteric and chiral smectic liquid crystals. Phys. Rev. E 1998, 58, 3264. [CrossRef]

12. Mikhailenko, V.; Krivoshey, A.; Pozhidaev, E.; Popova, E.; Fedoryako, A.; Gamzaeva, S.; Barbashov, V.; Srivastava, A.K.; Kwok, H.S.; Vashchenko, V. The nano-scale pitch ferroelectric liquid crystal materials for modern display and photonic application employing highly effective chiral components: Trifluoromethylalkyl diesters of p-terphenyldicarboxylic acid. J. Mol. Liquids 2019, 281, 186. [CrossRef]

(C) 2019 by the authors. Licensee MDPI, Basel, Switzerland. This article is an open access article distributed under the terms and conditions of the Creative Commons Attribution (CC BY) license (http://creativecommons.org/licenses/by/4.0/). 